\title{
OBSERVATIONS OF FLAME BEHAVIOR IN A LABORATORY-SCALE PRE-MIXED NATURAL GASIAIR GAS TURBINE COMBUSTOR FROM PLIF MEASUREMENTS OF OH
}

\author{
Paul O. Hedman, Thomas H. Fletcher, Stewart G. Graham, G. Wayne Timothy, \\ Daniel V. Flores, and Jason K. Haslam \\ Department of Chemical Engineering, Brigham Young University, Provo, Utah, USA 84602
}

\begin{abstract}
The objective of this study was to obtain instantaneous planar laser induced fluorescence (PLIF) images of $\mathrm{OH}$ in a laboratory-scale, gas-turbine combustor (LSGTC) with a premixed, swirl-stabilized, natural gas flame. Instantaneous PLIF images of $\mathrm{OH}$ were obtained at each of four operating conditions (high swirl and medium swirl at fuel equivalence ratios of 0.80 and 0.65). Comparison of the instantaneous images illustrates the stochastic nature of the flame structure. Pixel by pixel statistical analysis of each collection of images allowed both mean and standard deviation images to be generated, and analysis at selected locations has allowed probability density functions to be obtained in various regions of the flame structure. PLIF images of $\mathrm{OH}$, along with visual photographs and video recordings, showed a wide variation in flame structure for the different operating conditions. The variations in flame shapes are primarily a result of the effect of the swirl intensity and fuel equivalence ratio. Changes in the airflow rate over an order of magnitude do not seem to affect the visual flame structure in this experiment. Operation at $\phi=$ 0.80 produced the most stable flames with both injectors. The flame with the high swirl injector was more coalesced and closer to the injector than with the medium swirl injector. At $\phi$ $=0.65$, the flame was quite unstable for both swirl injectors. With the medium swirl injector, the flame would oscillate between two different flame structures, one that was more or less attached to the vortex funnel, and one that was lifted well above the vortex funnel. The MS case at $\phi=0.65$ was at the very edge of the lean flammability limit, and would on occasion extinguish.
\end{abstract}

\section{INTRODUCTION}

This study was part of a series of laser-diagnostic experiments performed on a common combustion apparatus. Companion studies were performed to measure gas velocities using two-component laser Doppler anemometry (LDA) [1] and to measure gas temperatures and concentrations using coherent anti-Stokes Raman spectroscopy (CARS) [2]. These data were obtained principally for evaluation of comprehensive gas turbine combustion models [3-6]. The work presented here is the result of planar laser induced fluorescence (PLIF) measurements of $\mathrm{OH}$ in a laboratory-scale, gas-turbine combustor (LSGTC) with a pre-mixed, swirl-stabilized, natural gas flame. $\mathrm{OH}$ is an important radical species in the hydrocarbon combustion process, and is thought to form at flame fronts. Therefore the spatial measurements of $\mathrm{OH}$ can be used to determine regions of combustion and the relative combustion intensity.

The LSGTC was operated at two swirl conditions and two fuel equivalence ratios $(0.80$ and 0.65$)$ at atmospheric pressure. This paper reports on the instantaneous, mean, and probability density functions (PDF's) of PLIF images of $\mathrm{OH}$ that were obtained with premixed natural gas/air in the LSGTC at these four different operating conditions.

Past experience at BYU/ACERC has shown that the modeling of combustion behavior is more accurate and proceeds more rapidly when coupled with pertinent, foundational experimental research. The LSGTC simulates many of the key combustor characteristics of commercial gas turbines [7], and provides a realistic flame where model predictions and in situ measurements can be compared. Use of advanced optical diagnostics permits near-instantaneous, non-intrusive measurements that provide insight into the physical processes that govern the combustion processes. The measurements also provide a database suitable for model sub-code evaluation and verification.

The objective of this part of the experimental program was to obtain sets of 256 instantaneous two-dimensional images of the $\mathrm{OH}$ radical using PLIF at each of four separate experimental conditions. Although qualitative, these instantaneous twodimensional images show the chaotic nature of the turbulent flame.

\section{COMBUSTOR TEST FACILITY}

The combustion facility used for this study is the same as that used by Hedman and coworkers [8-10]. Schmidt and Hedman [11] incorporated a premixed injector and used the burner to make CARS temperature and LDA velocity 
measurements on a premixed propane/air flame. The facility was further modified for this study to allow investigation of a premixed natural gas/air flame. This new premixed natural gas/air combustor has been designated the ATS Burner.

The actual combustion chamber, illustrated in Figure 1, was designed to reproduce the characteristics found in a modern annular combustor [7]. The combustor, which was provided to BYU by Wright Laboratory, Wright-Patterson Air Force Base [12], consists of a four-sided chamber with metal fillets in the corners to more nearly simulate an axisymmetric combustor while maintaining adequate optical access. Each of the four walls can be either a metal plate or a quartz window, depending on the optical access required. The premixed swirl injector, illustrated in Figure 2, is located in the center of the combustion chamber base. The injector consists of a stainless steel tube with a honeycombed brass insert. The brass insert serves to smooth the flow as well as provide a flame arrestor in case of flashback in the premixed fuel air mixture. Inserts are installed in the injector to provide different magnitudes of swirl to the inlet flow. The swirler inserts are placed above the brass honeycomb and secured by a threaded post that anchors the swirler to the brass honeycomb. A cap with an 18-mm hole in the center and beveled walls is placed on the top of the steel tube to coalesce the swirling flow at the injector exit. This cap was needed in order to make the burner operate successfully.

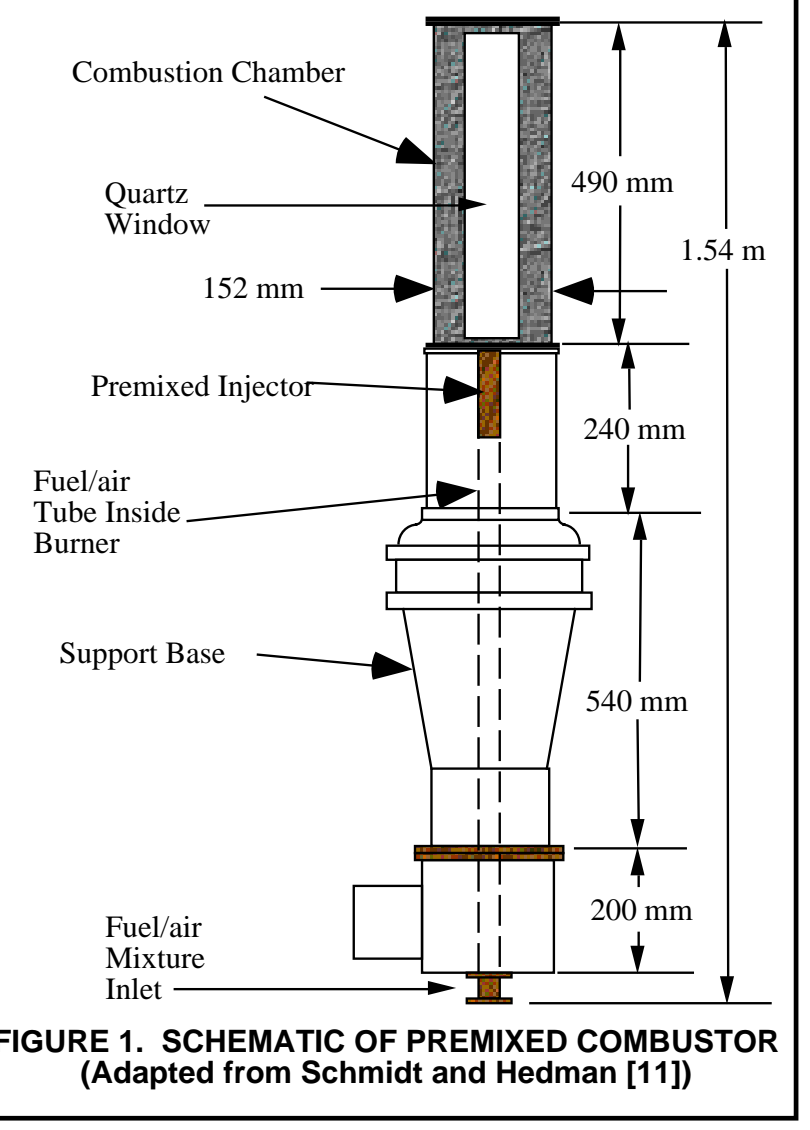

Figure 2 also shows a schematic of the two swirl inserts used in this study. The swirl inserts are labeled high swirl (HS) and medium swirl (MS) to coincide with the 60 and 45-degree slot angles. The swirl number (SN) indicated in Figure 2 is a nondimensional number that ratios the axial flux of tangential momentum to the axial flux of axial momentum times the equivalent nozzle radius [13]. A swirl number close to zero ( $\mathrm{SN}<<1)$ means the axial momentum is very large compared to the tangential momentum, whereas a very large swirl number $(\mathrm{SN}>>1)$ indicates that the axial momentum is small compared to the tangential momentum.

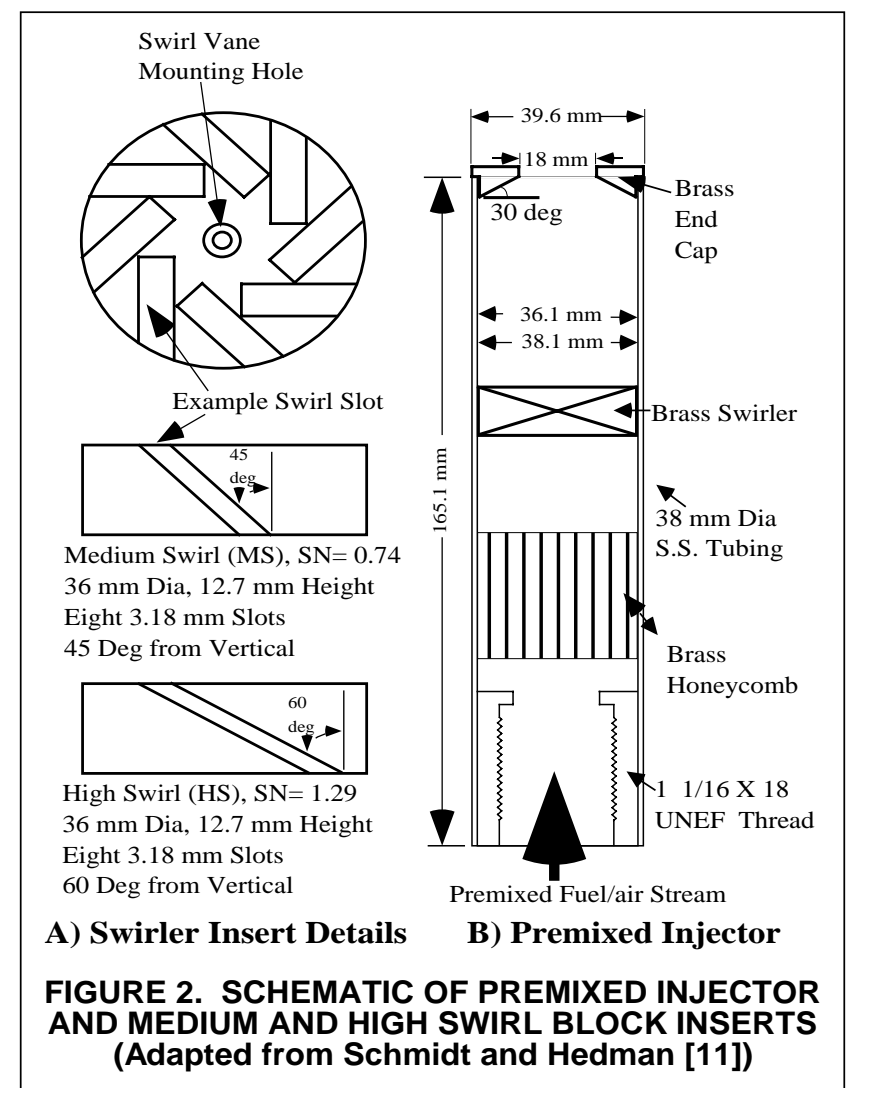

Fuel flow to the burner was measured with a variable area flow meter and is reported in standard liters per minute, or slpm, referenced to $1 \mathrm{~atm}, 70^{\circ} \mathrm{F}\left(21.1^{\circ} \mathrm{C}\right)$. The airflow to the burner was controlled by varying the pressure upstream of a choked flow nozzle. The diameter of the choked flow nozzle used for this study was $3.77 \mathrm{~mm}$. and provided airflow rates from 250 to $1000 \mathrm{slpm}$, depending on upstream pressure. Uniform mixing of gaseous fuel and air was assured by: (a) the length of the tube from the mixing point to the burner; (b) by a jet mixer in the feed system; and by (c) a honeycomb flame arrestor. The airflow rate for the four test conditions used in this study was $500 \mathrm{slpm}$.

\section{VISUAL FLAME CHARACTERIZATION}

Figure 3 presents a schematic drawing of the flow patterns present in the swirl-stabilized flame. The swirling flow creates a funnel or tornado-like vortex flow centered on the injector 
exit. A central recirculation zone and a side recirculation zone accompany the tornado-like flow. Both recirculation zones have been found to influence flame stability. Depending on swirl number and fuel equivalence ratio, one or the other of the recirculation zones may dominate the flame stability [1].

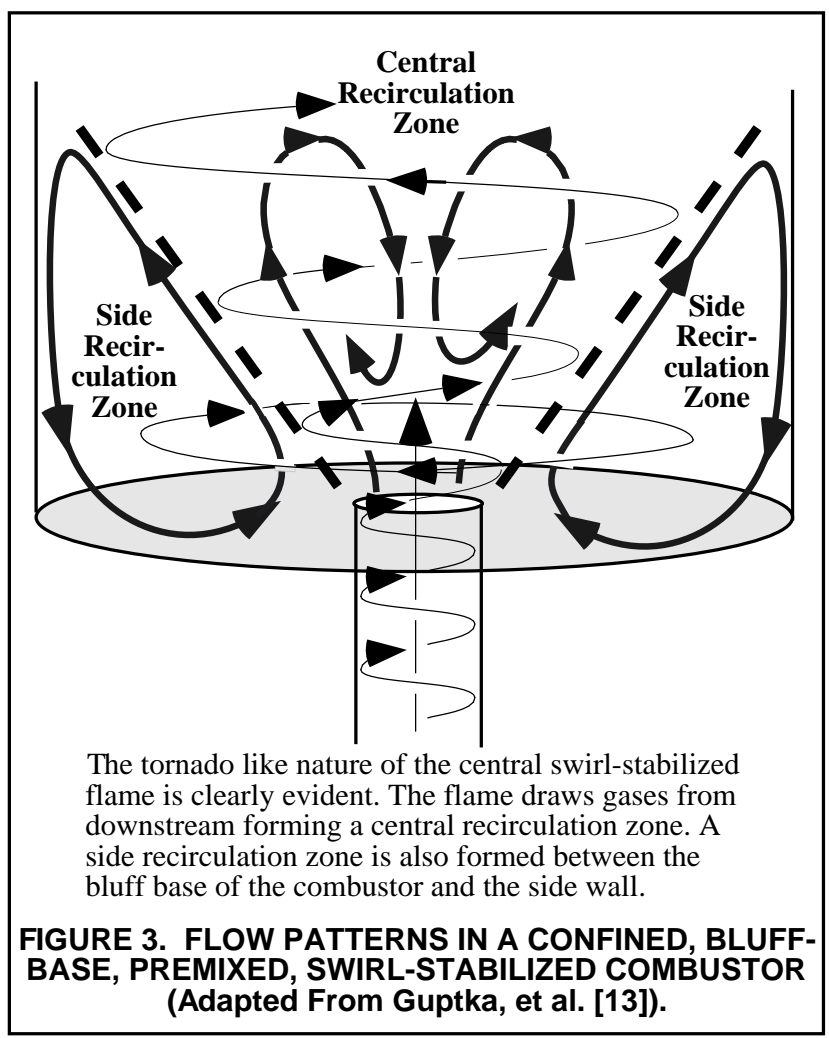

Wide variations in flame shape have been observed in the ATS Burner for different combinations of swirl number and fuel equivalence ratio. These structures were studied both visually, through photographic and video recordings, and with the PLIF images obtained in this study. Such observations have yielded insight into the location of the flame front, variation of flame structure with operating conditions and the presence and strength of the recirculation zones. These flame shapes are primarily a result of the effect of the swirl intensity and fuel equivalence ratio on the flow field, with the magnitude of the airflow rate being of secondary importance. The equivalence ratio was varied by changing the fuel flow rate while maintaining a constant 500-slpm air flow rate. The changes in flame shape were observed using the point of attachment, the location of the flame front, the structure of the vortex, and the visible intensity of the flame.

An example of a visual image of the flame structure is presented in Figure 4 for a stoichiometric $(\phi=1.0)$, medium swirl case. The $\phi=1.0$ image was included in the paper because this image was brighter, more visual, and reproduced better. The images at four test conditions (not included herein) are similar and show a small but significant effect of swirl number and fuel equivalence ratio even over the fairly narrow range of operating conditions (HS and MS injectors, and $\phi=$
0.80 and 0.65 ). Operation at wider limits in fuel equivalence ratio demonstrated wide variations in the observed flame structures. Nevertheless, the images from the four specified test conditions show significant variations with swirl number and $\phi$. Operation at $\phi=0.80$ produced the most stable flames. The flame with the high swirl injector was more coalesced and closer to the injector than with the medium swirl injector. At $\phi=0.65$, the flame was near the lean flammability limit, and was quite unstable for both swirl injectors. With the medium swirl injector, the flame would oscillate between two different flame structures, one that was more or less attached to the vortex funnel, and one that was lifted well above the vortex funnel. The MS case at $\phi=0.65$ was at the very edge of the lean flammability limit, and on occasion would actually extinguish.

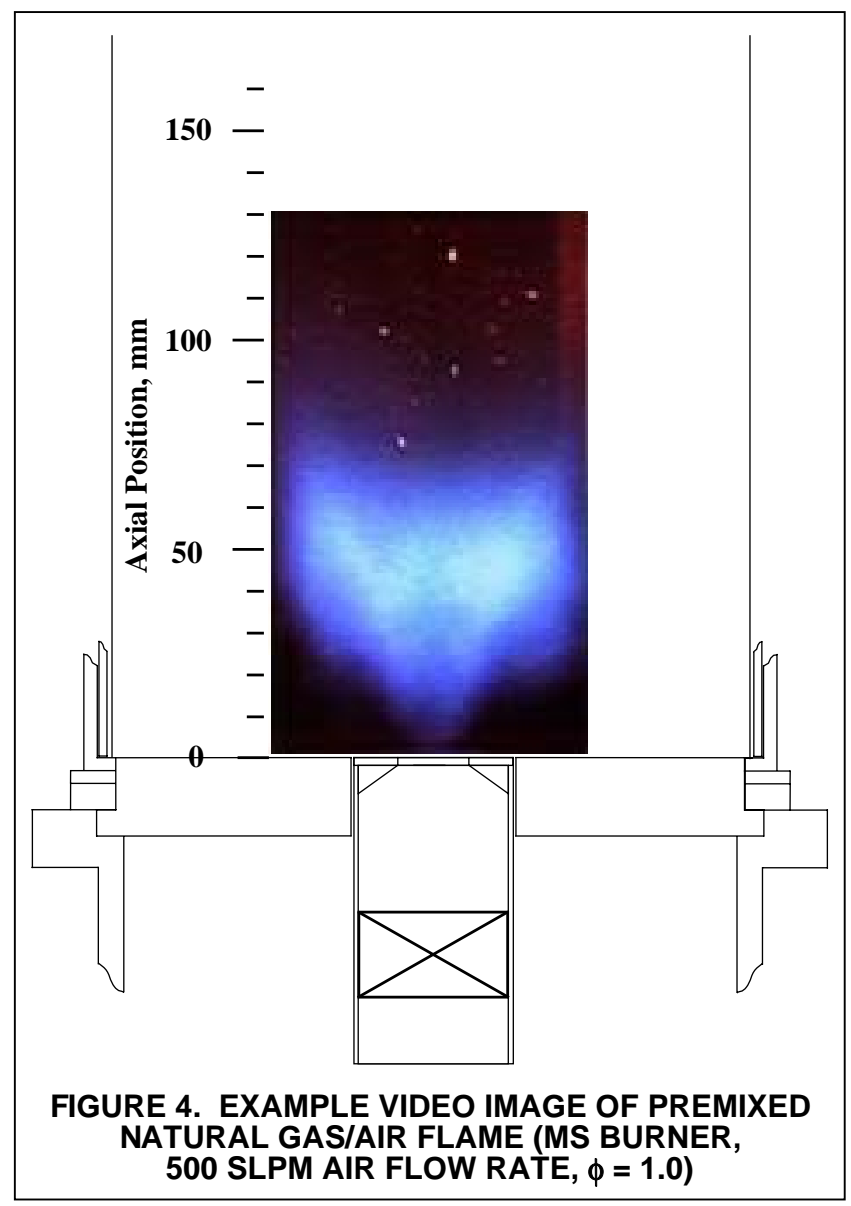

\section{PLIF APPARATUS}

Planar laser-induced fluorescence (PLIF) is a valuable tool for studying combustion phenomena. Flame propagation is partly governed by the diffusion and transport of important combustion intermediates like $\mathrm{OH}$. Laser-induced fluorescence has the potential of measuring trace amounts of these short lived combustion species at near instantaneous temporal resolution (10-100 ns). Likewise, because PLIF is laser-based, probing of the burner is non-intrusive. Laser-induced fluorescence measurements are often made at the focal point of a 
converging excitation laser [14]. However, due to the advances in detector and laser performance, fluorescence can be captured instantaneously over a two-dimensional area for many species. The two-dimensional area captured resembles an instantaneous photograph-like image of the concentration of the combustion species being probed. The flame is probed with a thin sheet of laser light tuned to the appropriate wavelength to cause fluorescence in the species of interest. The fluorescence occurs at a slightly different wavelength than that of the laser, and is detected at a location perpendicular to the laser sheet. The intensity of the fluorescence at each location in the laser sheet is directly proportional to the concentration of that species. The PLIF images provide detailed information regarding the location and shape of the flame structure. This structural information is very useful in analyzing burner designs, understanding combustion phenomena, and validating combustion computer models.

In this study, planar laser-induced fluorescence (PLIF) was employed to image the $\mathrm{OH}$ radical in the LSGTC with a turbulent, premixed, medium swirl natural gas/ air flame. A schematic of the PLIF installation on the LSGTC is presented in Figure 5. The excitation source used to pump the $\mathrm{OH}$ radical (ca $283 \mathrm{~nm}$ ) came from frequency doubling $566 \mathrm{~nm}$ laser light from a tunable dye laser (Spectra Physics PDL-3) with a $\mathrm{KD}^{*} \mathrm{P}$ crystal in the wave extender. The tunable dye laser used a frequency doubled $\mathrm{Nd}$ :YAG laser (Quantaray) as a pump source. The final $283 \mathrm{~nm}$ exiting laser beam was formed into a thin sheet approximately $0.5 \mathrm{~mm}$ thick and $13.0 \mathrm{~cm}$ wide. As the laser beam passed through the flame, an electronic camera (Princeton Instruments, ICCD-5765/RB-E) that uses an intensified charge coupled display (ICCD) with an electronic shutter speed of $100 \mathrm{~ns}$ was used to capture the induced fluorescence (ca $308 \mathrm{~nm}$ ) of $\mathrm{OH}$. The ICCD camera was setup orthogonal to the laser beam and was fitted with appropriate filters to block unwanted light and with a Nikon f/1.2 quartz lens that would pass the uv light. Captured images were digitally transferred to computer memory for subsequent analysis.

\section{PLIF RESULTS}

Figure 6 presents four example instantaneous $\mathrm{OH}$ images for each of the four test conditions. Instantaneous images were selected that represent: (A) a minimum intensity flame structure; (B) a near average intensity flame structure; and (C) and a maximum intensity flame structure. The fourth image (D) was selected and included because of its oddity. The computer processing of the counts from each pixel into the false color images presented is somewhat misleading. The color/brightness scale was normalized to the peak relative intensity for that image whether that value was 400 or 4000 . Nevertheless, the instantaneous structure is well represented.

The observations about flame structure noted from the visual images are confirmed with the instantaneous PLIF images. Operation at $\phi=0.80$ produced stable flames with both the HS and MS injectors (viz. Figure 6, Images B-101 and B-126). The flame with the high swirl injector was more coalesced and closer to the injector than that with the medium swirl injector. At $\phi=0.65$, the flame was near the lean flammability limit, and was quite unstable for both swirl injectors. As noted visually, the flame would oscillate between two different flame

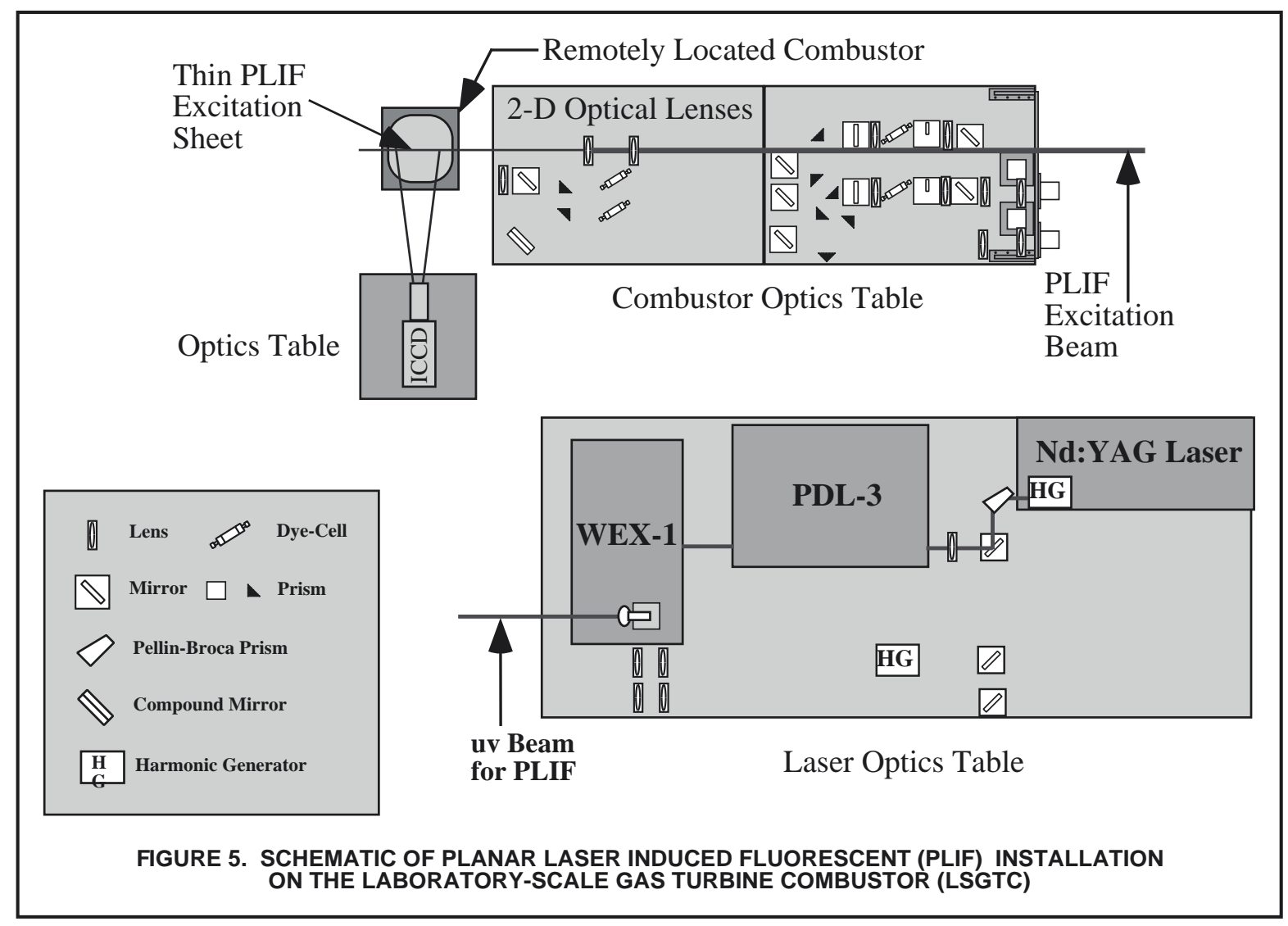



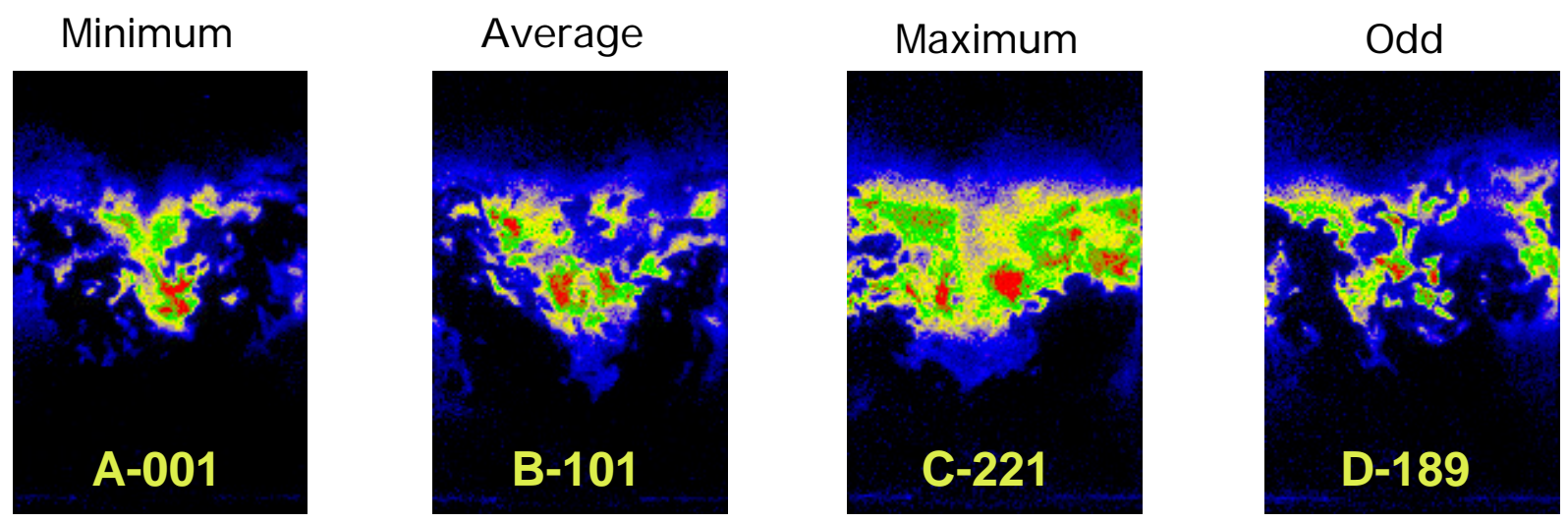

(A) Test Series 1, MS Injector, $\phi=\mathbf{0 . 8 0}$
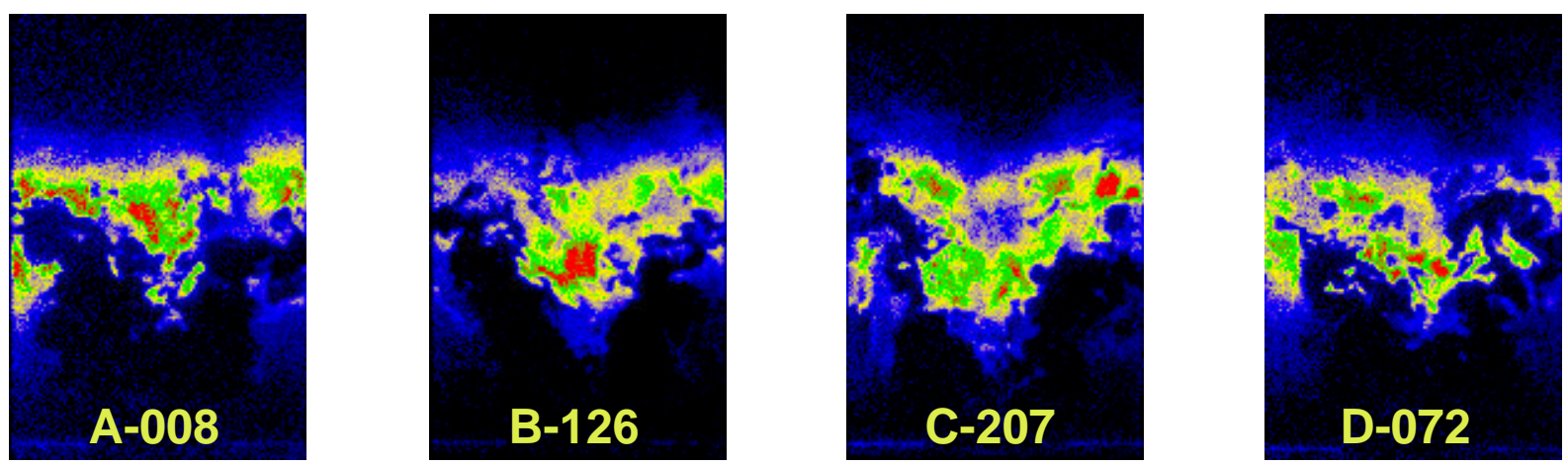

(B) Test Series 2, HS Injector, $\phi=\mathbf{0 . 8 0}$
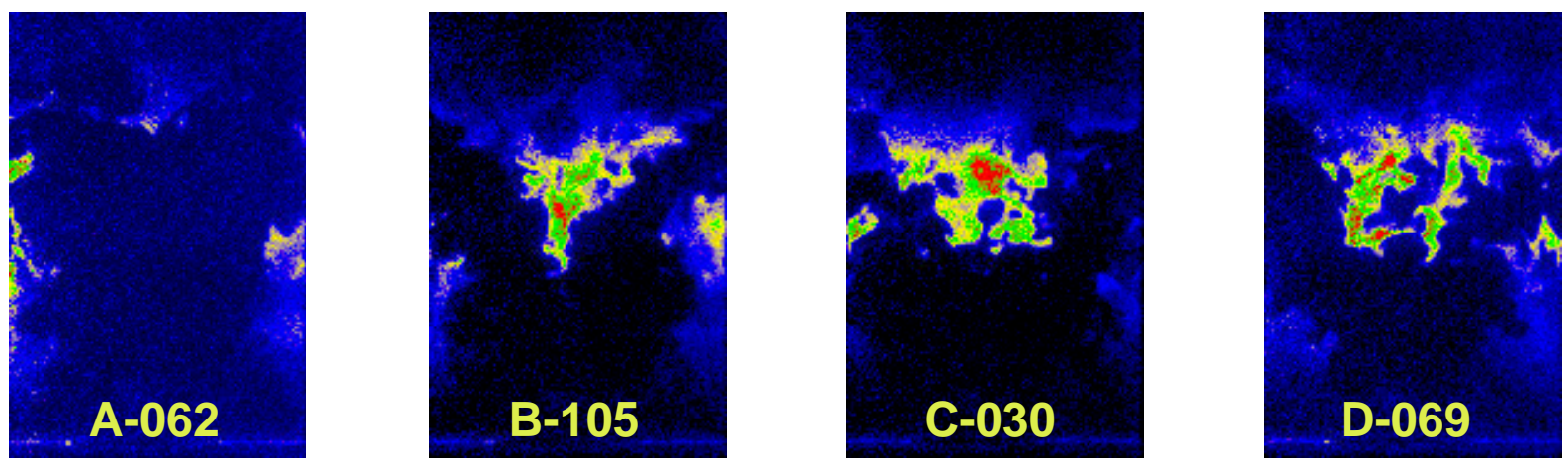

(C) Test Series 3, MS Injector, $\phi=0.65$
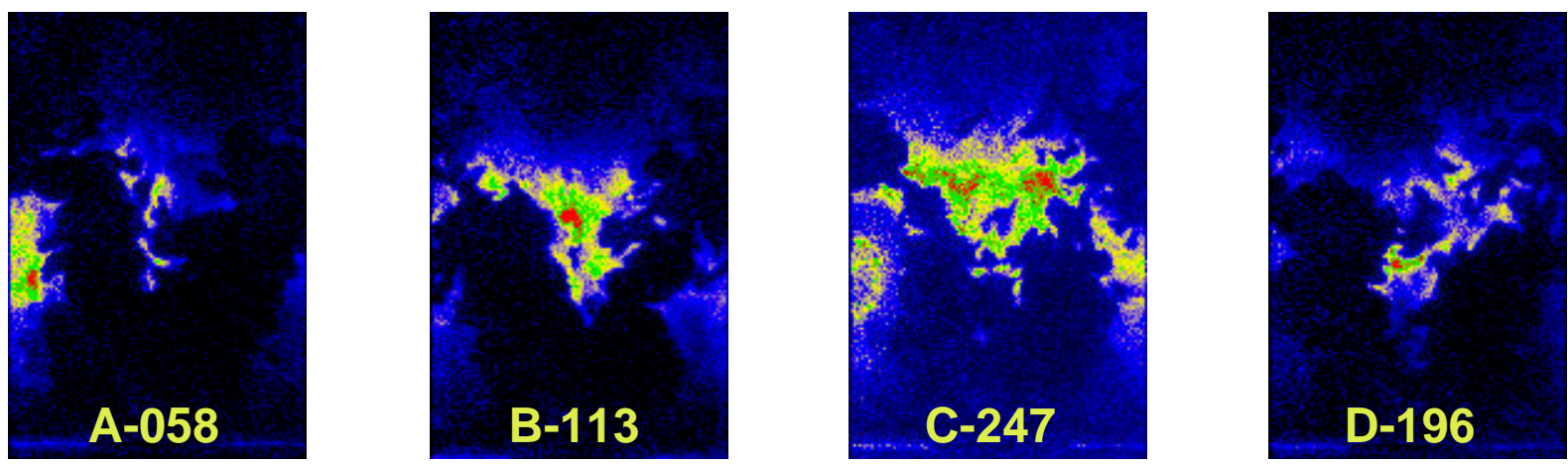

(D) Test Series 4, HS Injector, $\phi=0.65$

FIGURE 6. INSTANTANEOUS PLIF OH IMAGES OF FLAME STRUCTURE. 
structures, one that was more or less attached to the vortex funnel, and one that was lifted well above the vortex funnel. Visual observations showed that the MS case at $\phi=0.65$ was at the very edge of the lean flammability limit. Figure 6 , Image A-062, supports this observation, showing little $\mathrm{OH}$ in the central vortex and the flame being nearly extinguished. Even the image near the average structure (Figure 6, Image B105) shows little of the $\mathrm{OH}$ needed to support flame propagation.

The PLIF diagnostic capability allowed the collection of a significant number of separate instantaneous $\mathrm{OH}$ images (256 images at each test condition). Consequently, images of either the mean or the standard deviation in the fluorescence signal were obtained by applying the appropriate statistical analysis to the collection of images, pixel by pixel. Figure 7 presents the average image of the 256 separate laser shots for the $\mathrm{OH}$ species for the four test conditions. The mean image is very similar to the observed video images. However, the variation in pixel count, as reflected in the false color image, allows more insight into the structure of the flame and the character of the two different recirculation zones. As with the video images and instantaneous PLIF images, the mean PLIF images from the $\phi$ $=0.80$ cases indicate a stable flame, and very structured for both the HS and MS injectors. The vortex for the HS case is narrower, and the top of the flame zone is closer to the injector than in the MS case. The $\phi=0.65$ cases reflect a narrower vortex and lower flame zone for the HS configuration. Both configurations are near lean blowout at $\phi=0.65$, but the MS case is more unstable, and consequently more likely to extinguish than the HS case. Standard deviation PLIF images were also calculated pixel by pixel and are shown in Figure 8 for the four cases. The standard deviation gives insight into the amount of turbulence and ignition/extinction that occurs within the flame. The standard deviations in the HS conditions showed less spatial variation than in the corresponding MS conditions (viz. Figure 8, Images B and D compared to Images $\mathrm{A}$ and $\mathrm{C}$ respectively). The greater instability associated with the MS $\phi=0.65$ case is also apparent in Figure 8, Image C. As noted above, the false color transform from the detector counts has a different maximum value for each Image. These false color images therefore provide means for comparison of the shape of the flow field, as depicted by the standard deviation calculation, rather then the relative intensity.

Since the optical setup was identical for all test conditions, the actual detector counts may be proportional to the concentration of $\mathrm{OH}$ present at a given diagnostic location. [14] has suggested that in the linear regime the fluorescence signal power is proportional to the laser irradiance and the species population prior to laser excitation. However, the effects of saturation and quenching may cause the counts to be nonlinear. It is beyond the scope of this study to try to quantify this effect. However, the actual counts at selected pixel locations have been used to create PDFs within the flame structure. The number of average and peak counts, and the shape of the PDFs provide additional insights into the flame characteristics, and have provided a measure of the effect of operating condition on the relative level of $\mathrm{OH}$ in the flame.
Examples of PDF distributions for the most stable test case (HS, $\phi=0.80)$ and for the least stable test case (MS, $\phi=0.65$ ) are presented in Figures 9 and 10, respectively. Eight locations where the PDFs have been determined are superimposed on the mean PLIF image for both cases presented. The eight locations shown in Figures 9 and 10 are identical for both cases. Locations were selected above the flame, in the most intense regions of the flame, and below the flame. The PDF plots appear to be more similar to $\beta$-distributions rather than Gaussian distributions. In every case, the PDF shows that there is a significant amount of time that no $\mathrm{OH}$ exists at that location (e.g., Figure 9-D), even in the most intense part of the flame zone. Likewise, significant levels of $\mathrm{OH}$ are seen even in regions that are predominantly outside, but near the flame zone. In Figure 9 (HS, $\phi=0.80$ ) the maximum detector count (ca 4633 counts at maximum, 1652 counts at average) occurs at a location inside the intense part of the flame (Location D). In Figure 10, (MS, $\phi=0.65)$ the maximum detector count in the intense part of the flame (ca 1926 counts at maximum, 402 counts at average) occurs at location B. This suggests that the concentration of $\mathrm{OH}$ in the MS, $\phi=0.65$ case is about one fourth that of the HS, $\phi=0.80$ case. The non-linear PLIF response and the possibility of fluorescence saturation may alter the interpretation of concentration slightly. Nevertheless, the magnitude of the detector counts, as well as the corresponding PDF's, provide considerable insight into the character of the turbulent, reacting flame.

Figure 10 again further quantifies the unstable nature of the MS, $\phi=0.65$ case. Even in the most intense region (Location $\mathrm{B})$, there is a considerable amount of time when no $\mathrm{OH}$ is detected in the central vortex region. It is apparent that the stabilization process in the turbulent, swirl-stabilized flame is a very complicated process. The PLIF images obtained have provided some insight into this process, but should be combined with other data to provide a more complete understanding about the turbulent combustion process and flame stability.

\section{CONCLUSIONS}

A combustion chamber was designed to reproduce the characteristics found in a modern annular gas turbine combustor. This laboratory scale gas turbine combustor was used to investigate the characteristics of a premixed natural gas/air flame using PLIF at four operating conditions (HS and MS at $\phi=0.65$ and 0.80 ). Flame propagation is partly governed by the diffusion and transport of important combustion intermediates like $\mathrm{OH}$. Planar laser-induced fluorescence (PLIF) was therefore employed to image the $\mathrm{OH}$ radical in the LSGTC with a turbulent, premixed, medium swirl natural gas/ air flame.

Still photographs, video images, and PLIF images of $\mathrm{OH}$ showed a wide variation in flame shape for different combinations of swirl number and fuel equivalence ratio. The variations in flame shapes are primarily a result of the swirl intensity and fuel equivalence ratio on the flow field, with the magnitude of the airflow rate being of secondary importance. The observations about flame structure noted from the visual images were confirmed with the instantaneous PLIF images. 

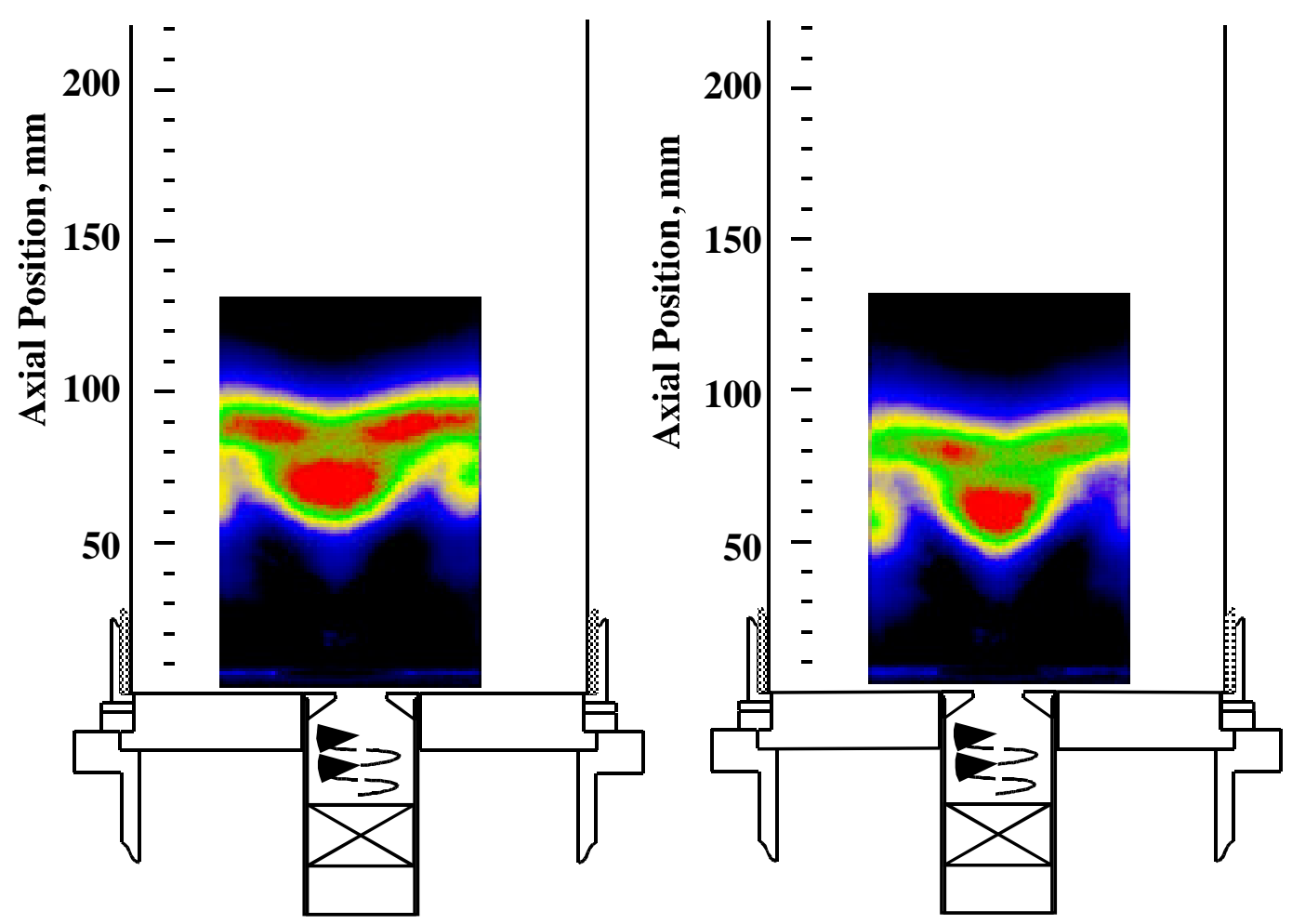

A) Medium Swirl, $\phi=0.80$

B) High Swirl, $\phi=0.80$
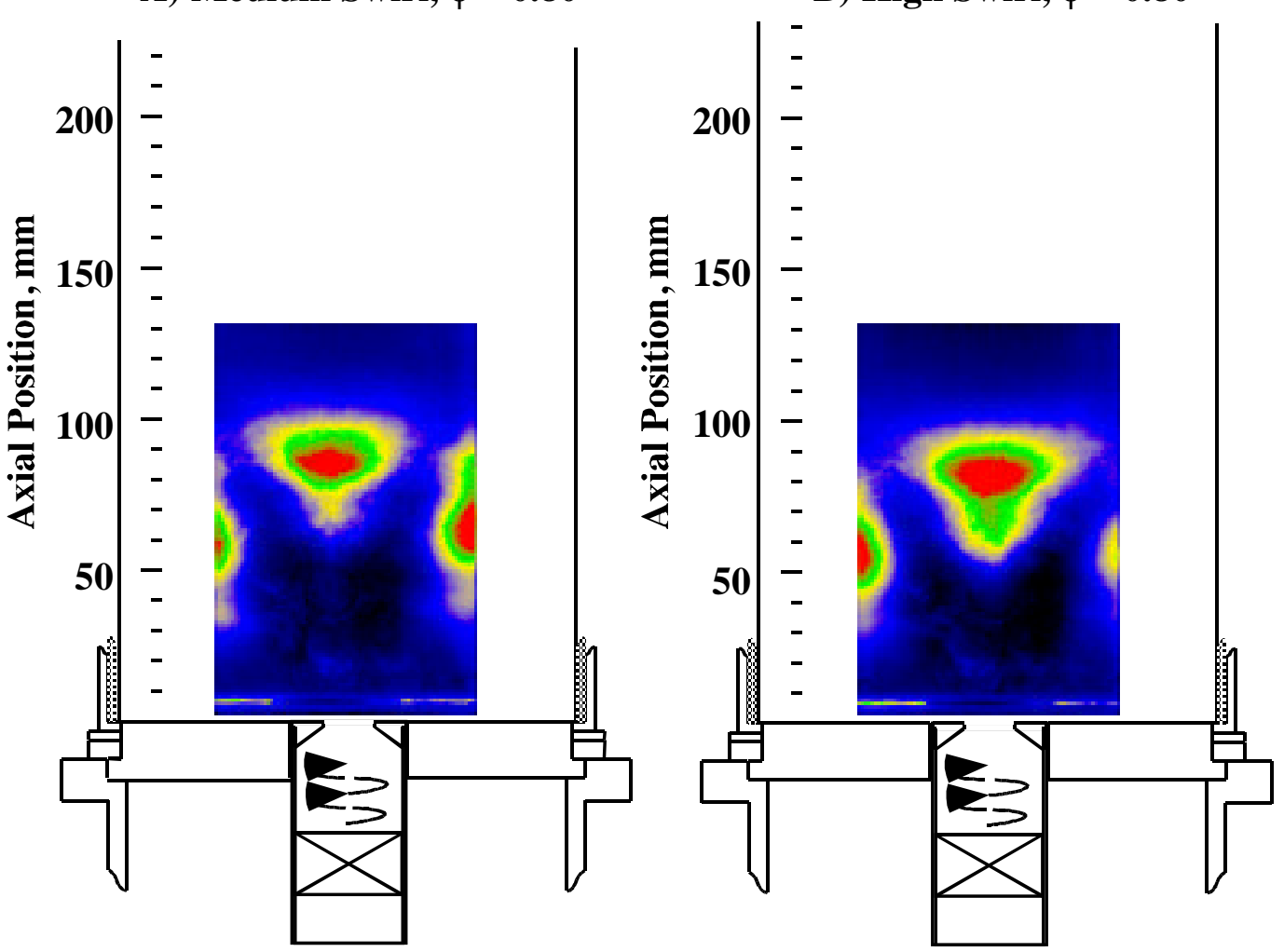

C) Medium Swirl, $\phi=0.65$

D) High Swirl, $\phi=0.65$

FIGURE 7. MEAN PLIF IMAGES OF OH IN THE ATS-BURNER (PREMIXED NATURAL GAS/AIR, AIR FLOW = 500 SLPM) 

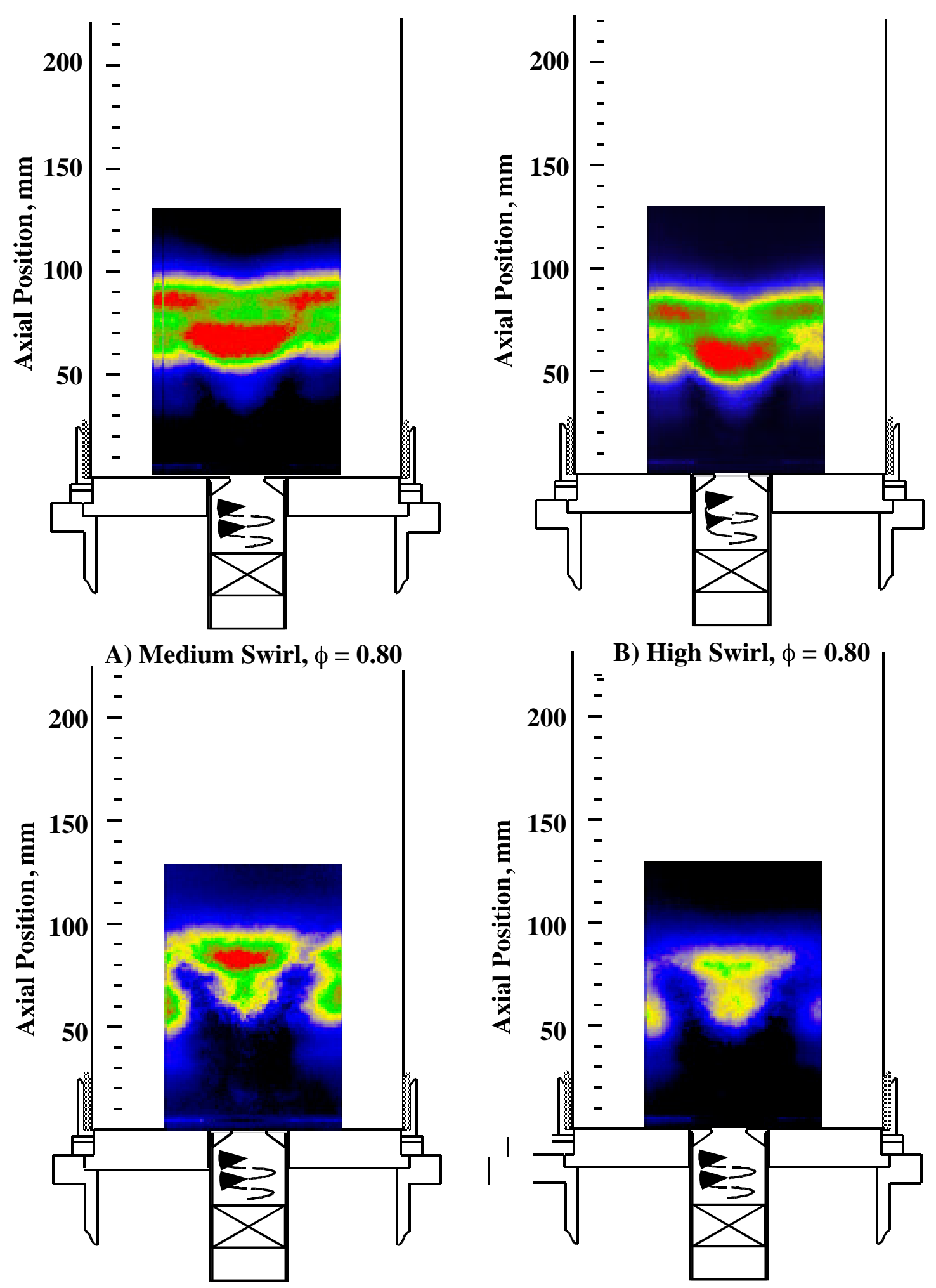

C) Medium Swirl, $\phi=\mathbf{0 . 6 5}$

D) High Swirl, $\phi=0.65$

FIGURE 8. STANDARD DEVIATION PLIF IMAGES OF OH IN THE ATS-BURNER (PREMIXED NATURAL GAS/AIR,AIR FLOW RATE = 500 SLPM) 


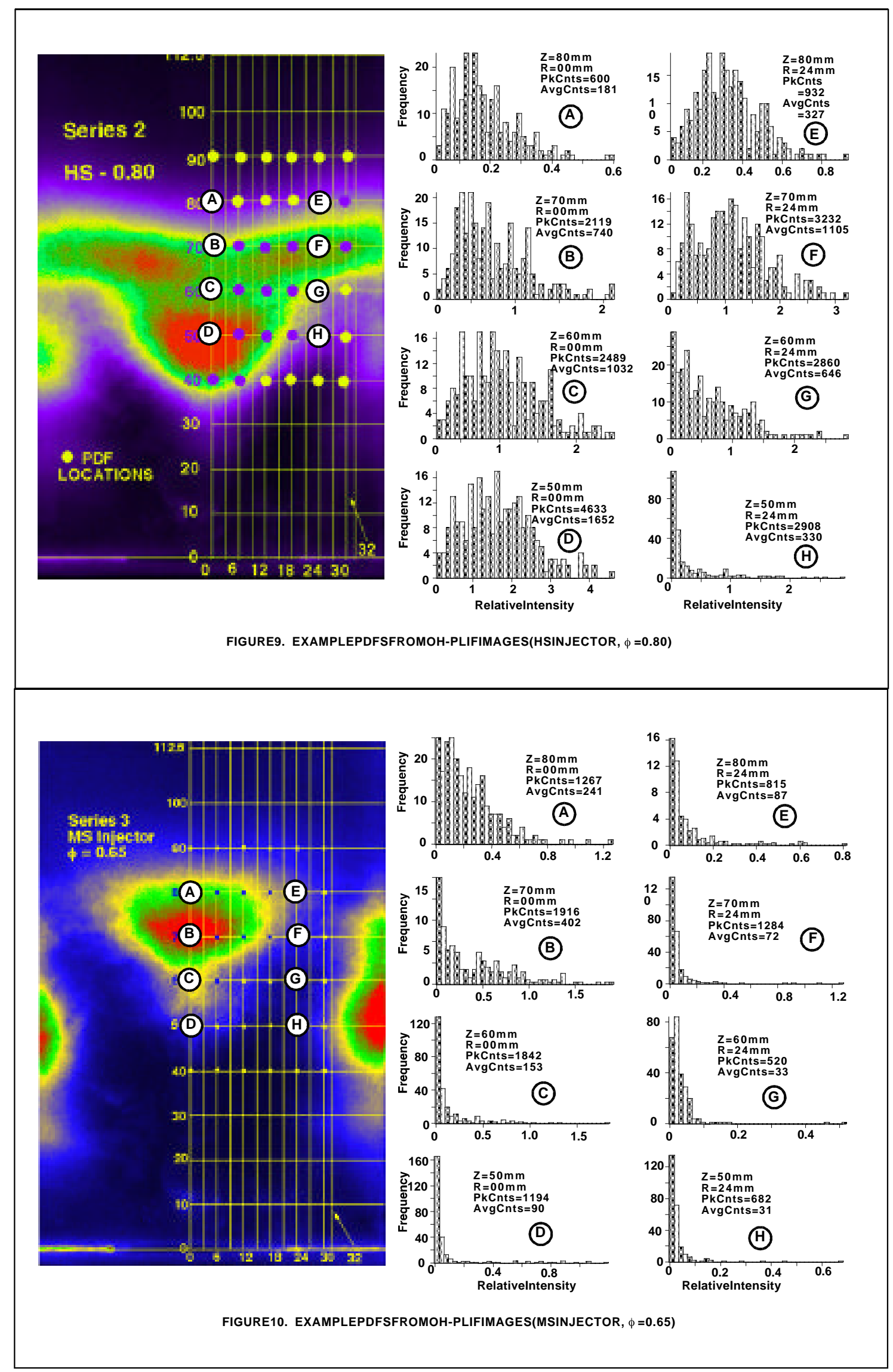


Operation at $\phi=0.80$ produced stable flames with both the HS and MS injectors. The flame with the high swirl injector was more coalesced and closer to the injector than that with the medium swirl injector. At $\phi=0.65$, the flame was near the lean flammability limit, and was quite unstable for both swirl injectors. The MS $\phi=0.65$ flame would oscillate between two different flame structures, one that was more or less attached to the vortex funnel, and one that was lifted well above the vortex funnel. The MS case at $\phi=0.65$ was at the very edge of the lean flammability limit, and on occasion would actually extinguish.

Mean and standard deviation of the OH PLIF image obtained from the 256 instantaneous images confirm the observations drawn from the visual and instantaneous PLIF images. The $\phi=$ 0.80 images are quite stable, and very structured for both the HS and MS injectors. The vortex for the HS case is narrower, and the top of the flame zone is closer to the injector than in the MS case. The $\phi=0.65$ cases reflect a narrower vortex and lower flame zone for the HS configuration. Both configurations are near lean blowout at $\phi=0.65$, but the MS case is more unstable, and consequently more likely to extinguish than the HS case.

The actual detector counts at selected pixel locations were used to create PDF's of $\mathrm{OH}$ intensity as a function of location in the flame structure. The maximum and average detector counts, along with the shape of the PDF's, provide interesting insights into the flame characteristics, and have provided a measure of the effect of operating condition on the relative level of $\mathrm{OH}$ in the flame. The PDF plots appear to be more similar to $\beta$-distributions rather than Gaussian distributions. The PDF plots show that there is a significant amount of time that no $\mathrm{OH}$ is detected in the most intense part of the flame zone. Likewise, significant levels of $\mathrm{OH}$ are seen even in regions that are predominately outside, but near the flame zone. The detector counts suggest that the maximum and average concentrations of $\mathrm{OH}$ for the $\mathrm{MS}, \phi=0.65$ case are about one fourth that of the HS, $\phi=0.80$ case.

The least intense PDF image for the MS, $\phi=0.65$ case supports the observation of great flame instability. In the most intense region of this flame, there was a considerable amount of time when there was essentially no $\mathrm{OH}$ present.

The PLIF images obtained have provided some insight into this process, but greater value can be obtained by combining these data with results from other related experiments (i.e., LDA and CARS).

\section{ACKNOWLEDGMENTS}

This paper presents results from a study that was funded in part by a U.S. Department of Energy's (DOE) Advanced Turbine Systems (ATS) program and with additional support by the Brigham Young University (BYU) Advanced Combustion Engineering Research Center (ACERC). This study was part of a more comprehensive effort by the U.S. Department of Energy's (DOE) Advanced Turbine Systems (ATS) program to develop advanced high efficiency gas turbines that demonstrated low pollution emissions.

\section{REFERENCES}

1. Murray, R. L., 1998, "Laser Doppler Anemometry Measurements in a Turbulent, Premixed, Natural Gas/Air Combustor," M. S. Thesis, Chemical Engineering Department, Brigham Young University, Provo, Utah. (See also Paper GT-2002-2053).

2. Flores, D. V., 2001, Ph.D. Dissertation, In Progress, Chemical Engineering Department, Brigham Young University, Provo, Utah 84602. (See also Paper GT-20022054).

3. Cannon, S. M., Brewster, B. S., and Smoot, L. D., 1998, "Stochastic Modeling of CO and NO in Premixed Methane Combustion," Combustion and Flame, Vol. 113, pp. 135146.

4. Cannon, S. M., Brewster, B. S., and Smoot, L. D., 1999, "PDF Modeling of Lean Premixed Combustion Using In Situ Tabulated Chemistry," Combustion and Flame, Vol. 119 , pp. 233-252.

5. Mallampalli, H. P., Fletcher, T. H., and Chen, J. Y., 1998, Evaluation of $\mathrm{CH}_{4} / \mathrm{NO}_{\mathrm{x}}$ Reduced Mechanisms Used for Modeling Lean Premixed turbulent Combustion of Natural Gas," ASME Journal of Engineering for Gas Turbines and Power, Vol. 120, pp. 703-712.

6. Meng, F. L., Brewster, B. S., and Smoot, L. D., 1997, "Comprehensive Model for Lean Premixed Combustion in Industrial Gas Turbines - Part II. Application," Spring Meeting of the Western States Section/The Combustion Institute, Sandia National Laboratories, Livermore, California.

7. Sturgess, G. J., Sloan, D. G., Lesmerises, A. L., Heneghan, S. P., and Ballal, D. R., 1992, "Design and Development of a Research Combustor for Lean Blowout Studies," ASME Journal of Engineering for Gas Turbines and Power, Vol. 114, pp. 13-19.

8. Hedman, P. O., Sturgess, G. J., Warren, D. L., Goss, L. P., and Shouse, D. T., 1995, "Observations of Flame Behavior from a Practical Fuel Injector Using Gaseous fuel in a Technology Combustor," ASME Journal of Engineering for Gas Turbines and Power, Vol. 117, pp. 441-452.

9. Hedman, P. O., and Warren, D. L., 1995, "Turbulent Velocity and Temperature Measurements from a Gas-Fueled Technology Combustor with a Practical Fuel Injector," Combustion and Flame, Vol. 100, pp. 185-192.

10. Warren, D. L., and Hedman, P. O., 1997, "Differential Mass and Energy Balances in the Flame Zone from a Practical Fuel Injector in a Technology Combustor," ASME Journal of Engineering for Gas Turbines and Power, Vol. 119, pp. 352-361.

11. Schmidt, S. E., and Hedman, P. O., 1985, "CARS Temperature and LDA Velocity Measurements in a Turbulent, Swirling, Premixed Propane/Air Fueled Model Gas Turbine Combustor," Paper Number 95-GT-64, ASME International Gas Turbine and Aeroengine Congress and Exposition, Houston, Texas (June 5-8).

12. Roquemore, W. M., 1992. Personal Communication, Wright Laboratory, Wright Patterson Air Force Base, Dayton, Ohio. 
13. Gupta, A. K., D. G. Lilley, and N. Syred, 1994, Swirl Flows, Abacus Press, Kent, United Kingdom.

14. Eckbreth, A. C., 1996, Laser Diagnostics for Combustion Temperature and Species, 2nd Edition, Gordon and Breach Publishers, Amsterdam, The Netherlands. 